\title{
Kinetic Study of Interaction between Solute Molecule and Surfactant Micelle
}

\author{
Kanji Miyabe, ${ }^{\dagger}$ Ryohei TaKahashI, and Youki ShimaZaKi \\ Department of Chemistry, Faculty of Science, Rikkyo University, 3-34-1 Nishi-Ikebukuro, Toshima, \\ Tokyo 171-8501, Japan
}

\begin{abstract}
We developed moment analysis of affinity kinetics by chromatographic capillary electrophoresis (MKCCE) method for the kinetic study of intermolecular interactions. Association and dissociation rate constants of the interaction in a micellar electrokinetic chromatography (MEKC) system between thymol and sodium dodecylsulfate micelle were determined by the MKCCE method. It is a method based on the moment theory for the kinetic study of intermolecular interactions under the conditions that neither immobilization nor chemical modification of molecules is required. In CCE mode, experimental conditions are controlled so that the migration of solute-micelle complex is stopped and only solute molecules migrate in a capillary. Mass transfer behavior of solute molecules in the CCE system is analogous to that in a chromatographic system. However, because it was difficult in practice to really perform CE experiments under the CCE conditions, CE data were measured with changing experimental conditions, i.e., applied pressure, under the conditions that the migration velocity of solute-micelle complex was around zero. The rate constants could be analytically determined from the CE data. In the MKCCE method, it is not necessary to fit elution curves numerically calculated to those experimentally measured for the determination of the rate constants. Regarding the interaction between thymol and SDS micelles, association equilibrium constant and association and dissociation rate constants were determined as $6.35 \times 10^{3} \mathrm{dm}^{3} \mathrm{~mol}^{-1}$, $5.6 \times 10^{4} \mathrm{dm}^{3} \mathrm{~mol}^{-1} \mathrm{~s}^{-1}$, and $8.7 \mathrm{~s}^{-1}$, respectively. It was demonstrated that the MKCCE method was effective for the kinetic study of intermolecular interactions.
\end{abstract}

Keywords Intermolecular interaction, affinity kinetics, moment theory, capillary electrophoresis, chromatographic capillary electrophoresis, association rate constant, dissociation rate constant, sodium dodecylsulfate, micelle, MEKC

(Received March 26, 2015; Accepted June 12, 2015; Published October 10, 2015)

\section{Introduction}

Surfactants and micelles have been used in analytical chemistry. ${ }^{1,2}$ Micellar electrokinetic chromatography (MEKC) is one of the examples. Surfactant micelles are used as quasistationary phases to improve the performance of capillary electrophoresis (CE). It is important to study the interaction between solute molecules and surfactant micelles from the kinetic point of view for well understanding the separation mechanism of MEKC.

$\mathrm{CE}$ has been used for analyzing intermolecular interactions. A number of papers have been published on the determination of the association equilibrium constant $\left(K_{\mathrm{A}}\right)$ between solute and ligand molecules. ${ }^{3-6}$ Some CE methods were also developed for the kinetic study of intermolecular interactions, ${ }^{7}$ e.g., affinity capillary electrophoresis, ${ }^{8}$ exponential decay method, ${ }^{9}$ and kinetic capillary electrophoresis (KCE).$^{10,11}$ In these methods, association rate constant $\left(k_{\mathrm{a}}\right)$ and dissociation rate constant $\left(k_{\mathrm{d}}\right)$ are determined by matching electropherograms or exponential decay curves experimentally measured to those calculated by changing the rate constants.

KCE was developed as a platform for kinetic homogeneous

$\dagger$ To whom correspondence should be addressed.

E-mail: kmiyabe@rikkyo.ac.jp affinity methods. ${ }^{12-14}$ In KCE, $k_{\mathrm{a}}$ and $k_{\mathrm{d}}$ were determined by fitting elution curves numerically calculated to experimental data. The macroscopic approach for studying kinetics at equilibrium (MASKE) was also developed for measuring equilibrium and kinetic parameters in the state of chemical equilibrium. ${ }^{15,16}$ It is required to tag a detectable label to solute or ligand molecules. It is a concern as to whether the chemical modification affects the analytical results of intermolecular interactions.

We developed moment analysis of affinity kinetics by chromatographic capillary electrophoresis (MKCCE) method as a new strategy for analyzing intermolecular interactions under homogeneous conditions in which both solute and ligand molecules are free in solution. No chemical modification, e.g., fluorescence labeling, is required for both solute and ligand molecules. In the CCE mode, experimental conditions of a CE system are controlled so that the migration of the solute-ligand complex in the longitudinal direction of a capillary is stopped. Only the solute molecules migrate and are detected. Mass transfer behavior of the solute molecules in the CCE system is analogous to that in a chromatographic system. It is expected that both the equilibrium and kinetic information about intermolecular interactions is obtained by the moment analysis from the elution peak profiles of the solute detected. We developed the moment equations, which were essential for determining $k_{\mathrm{a}}$ and $k_{\mathrm{d}}$ from elution peak profiles. However, 


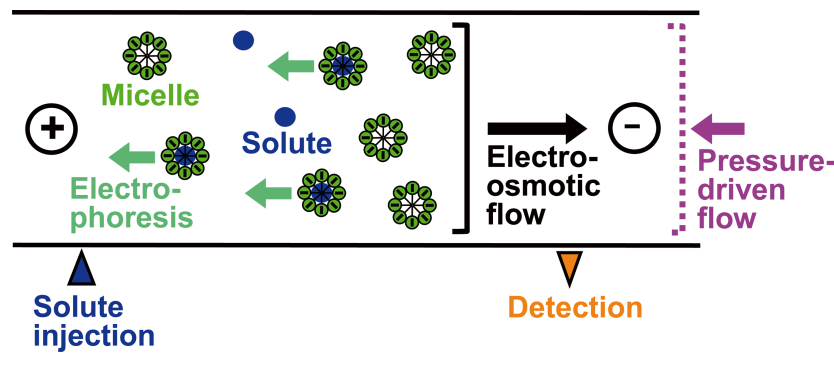

Fig. 1 Schematic illustration of the CCE system.

because it was difficult to really conduct CE experiments under the CCE conditions, we also developed practical experimental and data analysis procedures to determine $k_{\mathrm{a}}$ and $k_{\mathrm{d}}$ from electropherograms measured. CE data were measured with changing experimental conditions, i.e., applied pressure, under the conditions that the migration velocity of the solute-micelle complex was around zero. The MKCCE method was applied to a concrete MEKC system using sodium dodecylsulfate (SDS) micelles. Thymol was used as a neutral solute. The reaction rate constants $\left(k_{\mathrm{a}}\right.$ and $\left.k_{\mathrm{d}}\right)$ were analytically determined for the formation and dissociation of a thymol-SDS micelle complex. In the MKCCE method, it is not necessary to fit elution curves numerically calculated to those experimentally measured when the reaction rate constants are determined. It was demonstrated that the MKCCE method (CCE method with the moment theory) was effective for the kinetic study of intermolecular interactions.

\section{Theory}

\section{Principle of CCE}

The principle of CCE is explained by taking up the reaction in the MEKC system between thymol $(S)$ and SDS micelle $(L)$ to form a complex $(X)$ as one example, although the applicability of CCE is not limited to the system.

$$
S+L \underset{k_{d}}{\stackrel{k_{a}}{\rightleftarrows}} X
$$

where $k_{\mathrm{a}}$ and $k_{\mathrm{d}}$ are the rate constants for the association of $S$ and $L$ and for the dissociation of $X$, respectively. Figure 1 shows a schematic illustration of the CCE system, in which $S$ introduced at an injection port reacts with $L$ to form $X$. Because $S$ is neutral, $S$ is not electrophoresed. However, $X$ migrates toward the positive electrode because of its negative charge. Although $L$ is also electrophoresed in the same direction, it is assumed that the concentration of $L$ is constant because a run buffer containing SDS micelles is filled in the whole capillary. On the other hand, the run buffer itself migrates toward the negative electrode due to the electroosmotic flow (EOF). Because the velocity of EOF is faster than that of electrophoresis, not only $S$ but also $X$ migrates toward the negative electrode. Because $S$ is not electrophoresed, $S$ migrates faster than $X$ to the right direction in Fig. 1.

When a third flow having an appropriate direction and magnitude (a pressure-driven flow to the left direction in Fig. 1) is added under such conditions to the CE system, the migration of $X$ in the capillary is stopped as if $L$ is a stationary phase. When the third flow is generated by adding a pressure $\left(P_{\text {add }}\right)$, the migration of $X$ is just stopped at an appropriate applied pressure $\left(P_{\text {add }} *\right)$. Under such conditions, only $S$ migrates to the outlet of the capillary and is detected. This situation in the CCE system is analogous to that in an HPLC system. It is expected that both equilibrium and kinetic information about the intermolecular interaction between $S$ and $L$ can be analytically determined by the moment theory from the first absolute moment $\left(\mu_{1}\right)$ and second central moment $\left(\mu_{2}^{\prime}\right)$ of elution peaks. We call the CE system chromatographic capillary electrophoresis (CCE) for analyzing affinity kinetics between $S$ and $L$. We also developed the moment equations for determining $k_{\mathrm{a}}$ and $k_{\mathrm{d}}$ from $\mu_{1}$ and $\mu_{2}{ }^{\prime}$ of elution peaks.

However, it was difficult to actually perform MEKC experiments under the CCE conditions. Therefore, we also developed practical experimental and data analysis procedures to determine $k_{\mathrm{a}}$ and $k_{\mathrm{d}}$ from electropherograms experimentally measured. The practical experimental and data analysis procedures are explained in detail later.

\section{Moment equations}

The moment equations were developed for analyzing the elution peak profiles measured by the CCE experiments. The derivation procedure of the moment equations are explained in detail in Supporting Information. The equation for $\mu_{1}$ is formulated as follows.

$$
\mu_{1}-\frac{\tau}{2}=\frac{\int_{0}^{\infty} C e(t) t d t}{\int_{0}^{\infty} C e(t) d t}=\frac{Z}{u}\left(1+\frac{k_{\mathrm{a}}}{k_{\mathrm{d}}} C_{\mathrm{L}}\right)
$$

where $\tau$ is the width of rectangular solute injection pulse, $t$ the time, $C e(t)$ the function representing peak profile as a function of $t, Z$ the effective length of the capillary, $u$ the migration velocity of $S$, and $C_{\mathrm{L}}$ the concentration of $L$. Equation (2) indicates that $K_{\mathrm{A}}\left(=k_{\mathrm{a}} / k_{\mathrm{d}}\right)$ is obtained from $\mu_{1}$.

On the other hand, $\mu_{2}{ }^{\prime}$ consists of the contribution of axial diffusion of $S$ in the capillary and that of the reaction kinetics, i.e., association between $S$ and $L$ and dissociation of $X$.

$$
\begin{aligned}
\mu_{2}^{\prime}-\frac{\tau^{2}}{12} & =\frac{\int_{0}^{\infty} C e(t)\left(t-\mu_{1}\right)^{2} d t}{\int_{0}^{\infty} C e(t) d t} \\
& =\frac{Z}{u}\left\{\frac{2 D_{\mathrm{L}}}{u^{2}}\left(1+\frac{k_{\mathrm{a}}}{k_{\mathrm{d}}} C_{\mathrm{L}}\right)^{2}+\frac{2 k_{\mathrm{a}}}{k_{\mathrm{d}}^{2}} C_{\mathrm{L}}\right\}
\end{aligned}
$$

where $D_{\mathrm{L}}$ is the axial diffusion coefficient of $S$.

Both axial diffusion and migration of only $S$ are considered in Eqs. (2) and (3). Neither axial diffusion nor migration of $X$ is considered because the molecular weight of $X$ is sufficiently larger than that of $S$ and because the migration of $X$ is stopped under the CCE conditions. The molecular weight of SDS micelle is calculated as about $1.4 \times 10^{4}$ because that of SDS is 288 and an aggregation number of SDS micelle is about 49 (refer to Supporting Information). It is about two orders of magnitude larger than the molecular weight of thymol (150). It should be noted that Eqs. (2) and (3) are much simpler than the moment equations for chromatography because no separation media are packed in the capillary. ${ }^{17-21}$ The moment analysis procedure of experimental data is also much simpler in the case of CCE than HPLC.

Other moment equations were developed for $\mathrm{CE}$ systems, in which all the components, i.e., $S, L$, and $X$, simultaneously migrated in the capillary. ${ }^{22}$ However, it seems that they are not suitable for practical analysis of electropherograms experimentally measured because they are mathematically too complicated. It is probably difficult for many researchers to 
conveniently use them for determining $k_{\mathrm{a}}$ and $k_{\mathrm{d}}$. On the contrary, the introduction of the CCE concept makes the moment theory much simpler as Eqs. (2) and (3) because the migration of $X$ is stopped under the CCE conditions.

Equations (2) and (3) indicate how the intrinsic characteristics of the elution peak profile are correlated with several fundamental parameters of the association equilibrium, mass transfer rate, and reaction kinetics under CCE conditions. As defined, the values of $\mu_{1}$ and $\mu_{2}{ }^{\prime}$ were calculated from the whole profile of elution peaks experimentally measured, not from their specific parameters, e.g., position and width at half height. This means that $\mu_{1}$ and $\mu_{2}{ }^{\prime}$ represent intrinsic characteristics of the whole peak shape. The moment equations are derived with no assumption concerning the shape of elution peaks, e.g., the elution peak profile has to be represented by a Gaussian distribution curve.

\section{Experimental}

Experimental conditions and data analysis procedures are explained in detail in Supporting Information. They are briefly explained in the following. All experiments were conducted in a MEKC system containing SDS micelles by using a CE instrument with no special modification. An electrophoresis buffer was a mixture of an aqueous phosphate buffer $(20 \mathrm{mmol}$ $\mathrm{dm}^{-3}$ phosphate buffer of $\left.\mathrm{pH}=7.1\right)$ and methanol $(80 / 20, \mathrm{v} / \mathrm{v})$, which contains SDS at $15.4 \mathrm{mmol} \mathrm{dm}^{-3}$. Thymol and dimethylsulfoxide (DMSO) were used as solute and EOF maker, respectively. ${ }^{23-27}$ Thymol was dissolved with the mixture at $2.0 \mathrm{mmol} \mathrm{dm}{ }^{-3}$ to prepare a solute solution. Five microliters of DMSO was also dissolved in $1.5 \mathrm{~mL}$ of the solute solution. The solute solution was injected into the capillary by a pressure pulse $(0.5 \mathrm{psi})$ for $5.0 \mathrm{~s}$. Electrophoresis was carried out at electric field strength of $370 \mathrm{~V} \mathrm{~cm}^{-1}$. CE experiments were conducted with changing $P_{\text {add }}$ at $-0.1,0$, and +0.1 psi. Information about $\mu_{1}$ of thymol peaks was analyzed to determine the appropriate pressure $\left(P_{\text {add }} *\right)$, at which the migration of the solute-micelle complex in the longitudinal direction of the capillary is just stopped. The values of $k_{\mathrm{a}}$ and $k_{\mathrm{d}}$ were determined by the moment method from $\mu_{1}$ and $\mu_{2}^{\prime}$ of CE peaks of thymol and DMSO at $P_{\text {add }}$.

\section{Results and Discussion}

\section{Elution peak profiles}

Figure 2 illustrates elution peak profiles. The capillary was additionally pressurized during the $\mathrm{CE}$ experiments. Because of the specification of the CE instrument used, it was actually difficult to delicately adjust the magnitude of $P_{\text {add }}$. Because $P_{\text {add }}$ could be adjusted with the minimum increment of $0.1 \mathrm{psi}, P_{\text {add }}$ was changed at $-0.1,0$, and $+0.1 \mathrm{psi}$. The negative and positive symbols mean that the direction of the additional pressuredriven flow is opposite to and the same as the migration direction of $X$, respectively. The migration speed of $X$ to the outlet of the capillary is slower at $P_{\text {add }}=-0.1$ psi and faster at $P_{\text {add }}=+0.1 \mathrm{psi}$ than at $P_{\text {add }}=0$ psi.

It is referable for accurately determining $k_{\mathrm{a}}$ and $k_{\mathrm{d}}$ that the elution peak profiles are not too complicated. The elution time of thymol is longer in the order of $P_{\text {add }}=-0.1,0$, and $+0.1 \mathrm{psi}$. Accordingly, the peak of thymol is broader in the same order. Similar situations are also observed for the elution peaks of DMSO. On the other hand, the elution time of thymol is longer than that of DMSO. The peak width of thymol is also wider

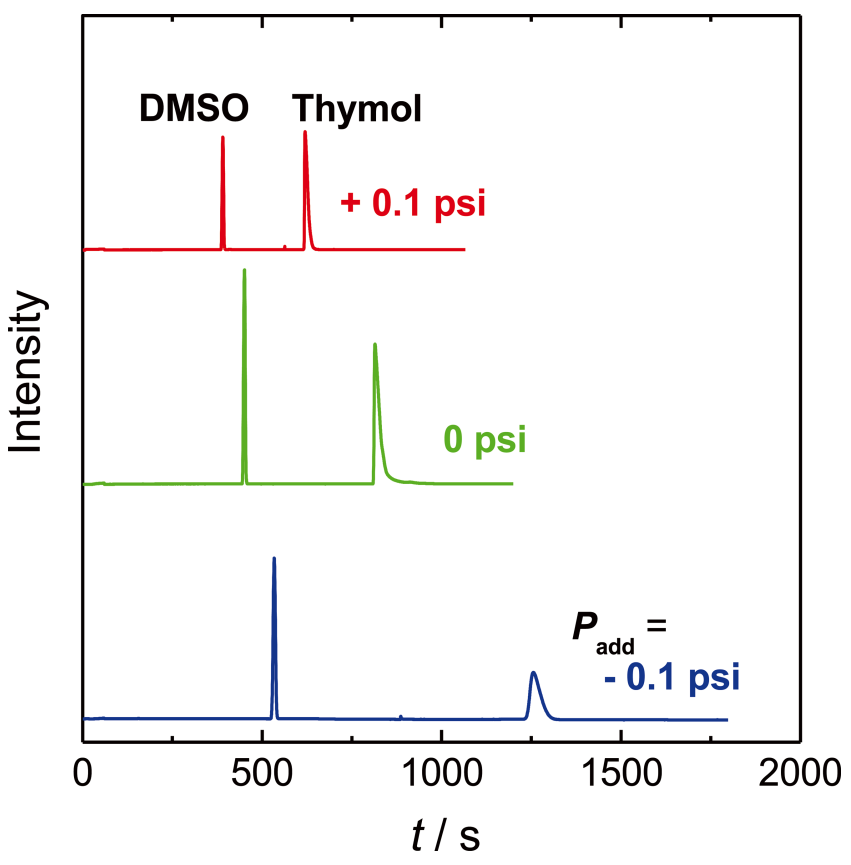

Fig. 2 Elution peaks of thymol and DMSO measured with SDS micelle.

than that of DMSO. These observations result from the interaction of thymol with SDS micelle. DMSO does not react with SDS micelle. The difference in the position and broadening of the elution peaks between thymol and DMSO was analyzed by the moment theory to determine $k_{\mathrm{a}}$ and $k_{\mathrm{d}}$ of the interaction between thymol and SDS micelle.

\section{Correlation of $\mu_{1}$ and $\mu_{2}{ }^{\prime}$ with $P_{\text {add }}$}

As explained in the Supporting Information, the influence of the length of solute injection pulse and the width of detection window on the experimental values of $\mu_{1}$ and $\mu_{2}^{\prime}$ was corrected to obtain their net values, although their influence was negligibly small. Their net values are plotted against $P_{\text {add }}$ in Figs. 3 and 4. The relative standard deviation for six iterative measurements of CE peaks of thymol and DMSO was $1.2-3.0 \%$ and $0.4-6.5 \%$ for $\mu_{1}$ and $\mu_{2}{ }^{\prime}$, respectively. As indicated in Figs. 3 and $4, \mu_{1}$ is linearly correlated with $P_{\text {add. }}$. This means that the CE system was accurately pressurized with the same increment of $P_{\text {add }}, e . g$., $-0.1,0$, and 0.1 psi.

\section{Estimation of $P_{\text {add }} *$}

The value of $P_{\text {add }} *$ was calculated from those of $\mu_{1}$ measured under the three different conditions of $P_{\text {add }}$. The average migration velocity of $S\left(v_{\text {Sav }}\right)$ in CE systems is represented as follows.

$$
v_{\mathrm{Sav}}=v_{\mathrm{S}} \frac{C_{\mathrm{S}}}{C_{\mathrm{S}}+C_{\mathrm{X}}}+v_{\mathrm{X}} \frac{C_{\mathrm{X}}}{C_{\mathrm{S}}+C_{\mathrm{X}}}=v_{\mathrm{S}} \frac{1}{1+K_{\mathrm{A}} C_{\mathrm{L}}}+v_{\mathrm{X}} \frac{K_{\mathrm{A}} C_{\mathrm{L}}}{1+K_{\mathrm{A}} C_{\mathrm{L}}}(4)
$$

where $C_{\mathrm{S}}$ and $C_{\mathrm{X}}$ are respectively the concentration of $S$ and $X$, $v_{\mathrm{S}}$ and $v_{\mathrm{X}}$ the migration velocity of $S$ and $X$, respectively. The value of $\mu_{1}$ is represented as follows.

$$
\mu_{1}=\frac{Z}{v_{\mathrm{Sav}}}=\frac{Z}{v_{\mathrm{S}}+v_{\mathrm{X}} K_{\mathrm{A}} C_{\mathrm{L}}}\left(1+K_{\mathrm{A}} C_{\mathrm{L}}\right)
$$

The second term in the denominator represents the influence of 


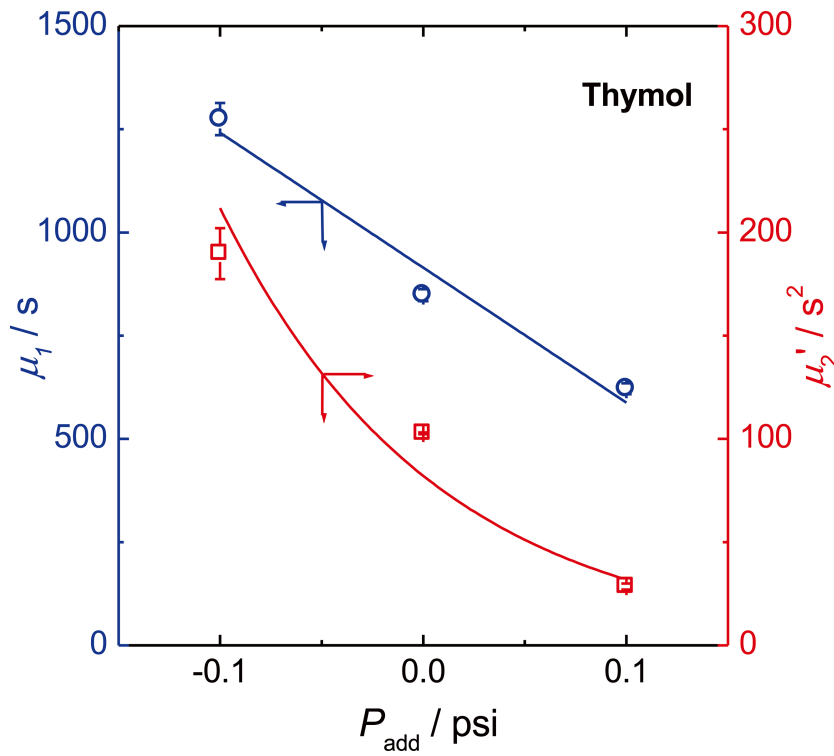

Fig. 3 Correlation between $\mu_{1}$ and $\mu_{2}^{\prime}$ of elution peaks of thymol with $P_{\text {add }}$.

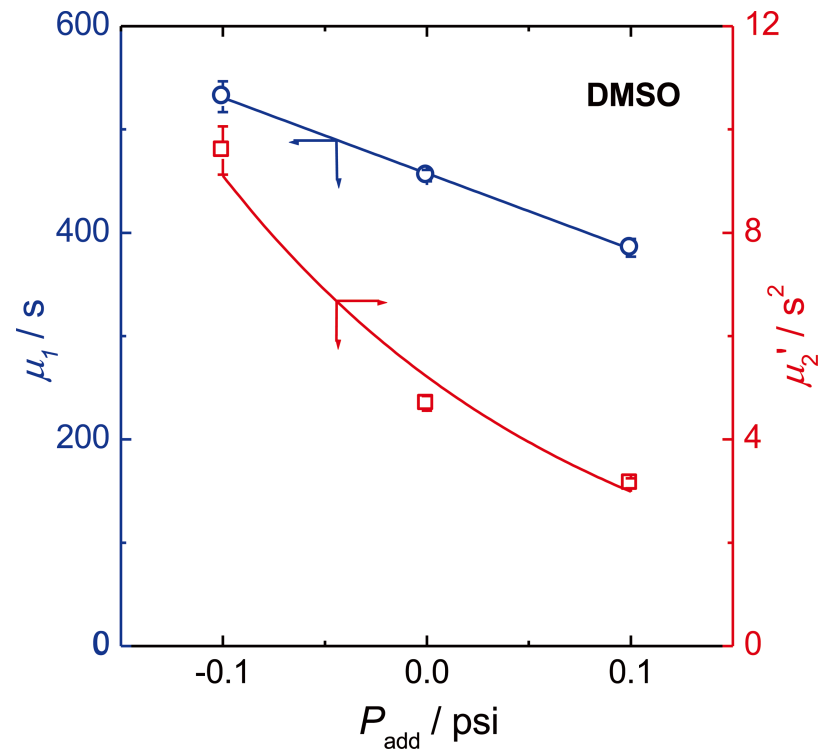

Fig. 4 Correlation between $\mu_{1}$ and $\mu_{2}{ }^{\prime}$ of elution peaks of DMSO with $P_{\text {add. }}$.

the migration velocity of $X$ on $\mu_{1}$. Equation (5) is the same as Eq. (2) under the CCE conditions because $X$ is stopped in the capillary and only $S$ migrates. Figure 5 indicates that $v_{\mathrm{S}}$ is linearly correlated with $P_{\text {add }}$. Because thymol is neutral, $v_{\mathrm{S}}$ is equal to the migration velocity of DMSO, i.e., EOF velocity, which was calculated from $\mu_{1}$ of DMSO. Figure 6 illustrates the correlation between $\mu_{1}$ of thymol and the reciprocal of $v_{\mathrm{S}}$ (open circles). Figure 6 indicates that a correlative straight line of the three open circles does not pass through the origin because $v_{\mathrm{X}}$ is not equal to zero.

In this study, $P_{\text {add }} *$ was determined by a successive approximation method. As a first step, a linear correlation passing through the origin (dashed line) was assumed in Fig. 6 by neglecting the influence of $v_{\mathrm{X}} K_{\mathrm{A}} C_{\mathrm{L}}$ on $\mu_{1}$ of thymol to calculate a first estimate of $K_{\mathrm{A}}$. It was calculated as $5.15 \times$

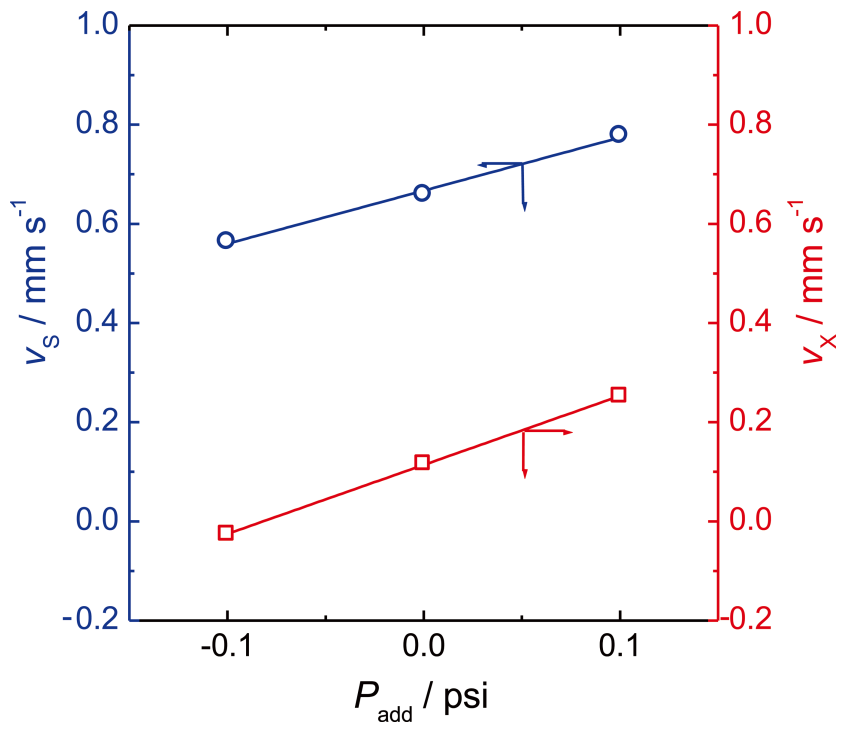

Fig. 5 Correlation of $v_{\mathrm{S}}$ and $v_{\mathrm{X}}$ with $P_{\text {add. }}$.

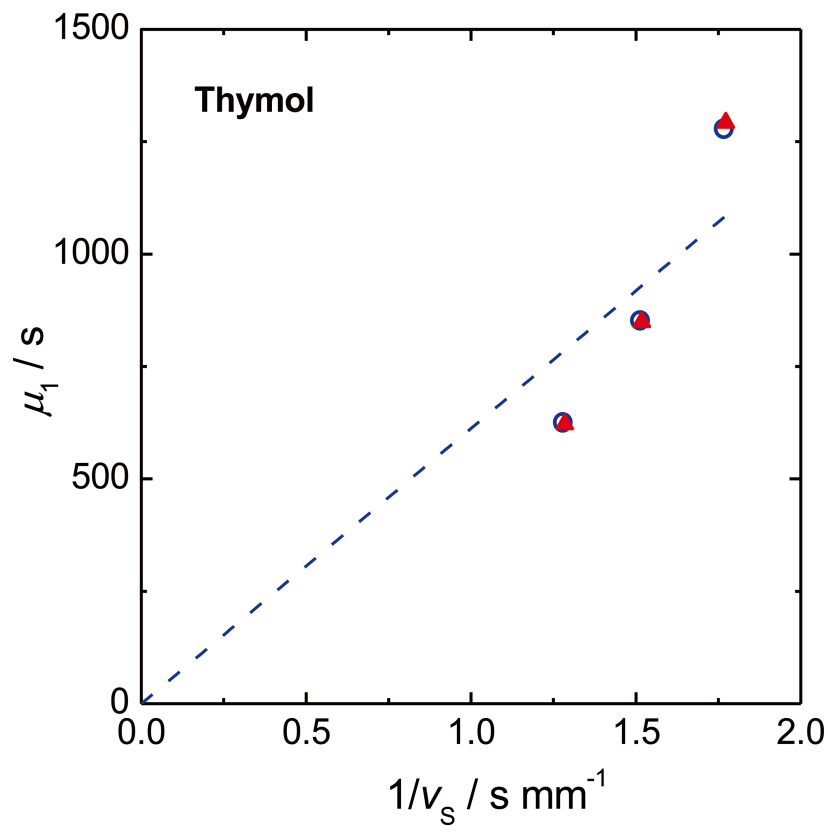

Fig. 6 Correlation between $\mu_{1}$ of thymol and the reciprocal of $v_{\mathrm{s}}$. Circles: the values of $\mu_{1}$ of thymol experimentally measured under the three conditions of Padd. Triangles: the values of $\mu_{1}$ of thymol calculated from the final values of $K_{\mathrm{A}}, \mu_{1}$, and $P_{\text {add }}{ }^{*}$.

$10^{3} \mathrm{dm}^{3} \mathrm{~mol}^{-1}$ from the slope of the dotted line in Fig. 6 on the assumption that $K_{\mathrm{A}}$ was constant irrespective of $P_{\text {add }}$ As illustrated in Figs. 3 and 5, $\mu_{1}$ and $v_{\mathrm{S}}$ of thymol change due to the change in $P_{\text {add. }}$. This means that three values of $v_{\mathrm{X}}$ at each $P_{\text {add }}$ are calculated from the $\mu_{1}$ and $v_{\mathrm{S}}$ data by Eq. (5) because all parameters except $v_{\mathrm{X}}$ are obtained.

The first solid line in Fig. 7 indicates the correlation between Padd and $v_{\mathrm{X}}$, which was calculated by Eq. (5) from the first estimate of $K_{\mathrm{A}}\left(5.15 \times 10^{3} \mathrm{dm}^{3} \mathrm{~mol}^{-1}\right)$. Then, first estimate of $P_{\text {add }} *$ was calculated as -0.040 psi from the intercept of the first solid line on the horizontal axis in Fig. 7. The value of $\mu_{1}$ at the first estimate of $P_{\text {add }} *$ is calculated as $1.05 \times 10^{3} \mathrm{~s}$ from Fig. 3 . 


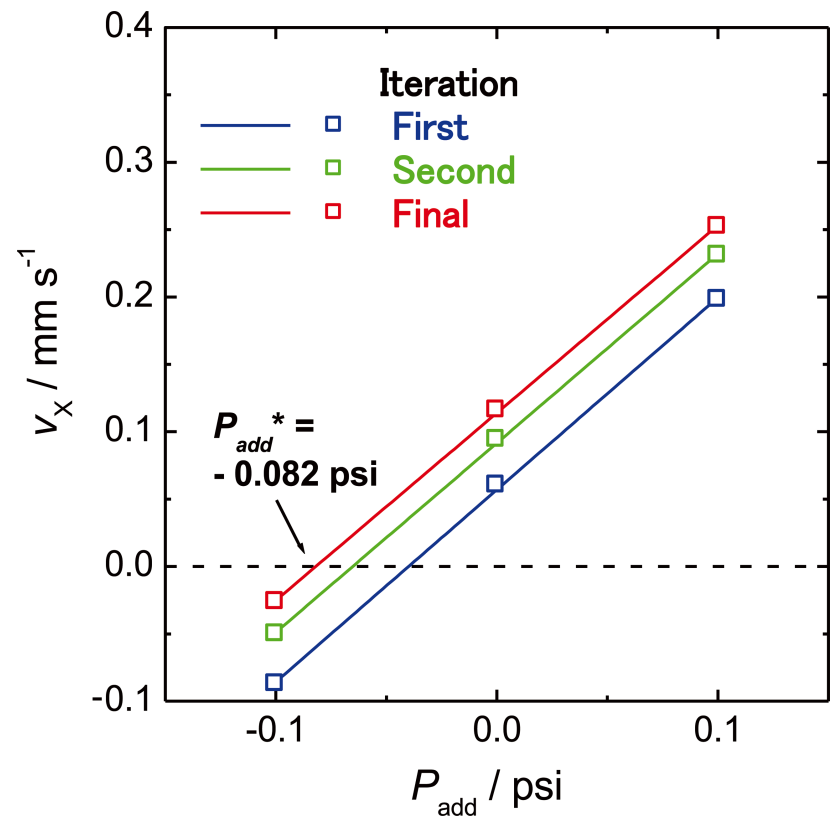

Fig. 7 Correlation between $v_{\mathrm{X}}$ and $P_{\text {add }}$.

Table 1 Successive approximation analysis for determining $K_{\mathrm{A}}$, $\mu_{1}$, and $P_{\text {add }} *$

\begin{tabular}{ccccc}
\hline Iteration & $\begin{array}{c}\text { Expected } K_{\mathrm{A}} \\
\mathrm{dm}^{3} \mathrm{~mol}^{-1}\end{array}$ & $\begin{array}{c}P_{\text {add }} * \\
\mathrm{psi}\end{array}$ & $\begin{array}{c}\mu_{1} \\
\mathrm{~s}\end{array}$ & $\begin{array}{c}\text { Resulting } K_{\mathrm{A}} \\
\mathrm{dm}^{3} \mathrm{~mol}^{-1}\end{array}$ \\
\hline 1 & $5.15 \times 10^{3} \longrightarrow$ & -0.040 & $1.05 \times 10^{3} \longrightarrow$ & $5.82 \times 10^{3}$ \\
2 & $5.82 \times 10^{3} \longrightarrow$ & -0.065 & $1.13 \times 10^{3} \longrightarrow$ & $6.17 \times 10^{3}$ \\
3 & $6.17 \times 10^{3} \longrightarrow$ & -0.076 & $1.16 \times 10^{3} \longrightarrow$ & $6.29 \times 10^{3}$ \\
4 & $6.29 \times 10^{3} \longrightarrow$ & -0.080 & $1.18 \times 10^{3} \longrightarrow 6.34 \times 10^{3}$ \\
5 & $6.34 \times 10^{3} \longrightarrow$ & -0.081 & $1.18 \times 10^{3} \longrightarrow 6.35 \times 10^{3}$ \\
6 & $6.35 \times 10^{3} \longrightarrow$ & -0.082 & $1.18 \times 10^{3} \longrightarrow$ & $6.35 \times 10^{3}$ \\
\hline
\end{tabular}

The value of $K_{\mathrm{A}}$ corresponding to the $\mu_{1}$ value was calculated as $5.82 \times 10^{3} \mathrm{dm}^{3} \mathrm{~mol}^{-1}$ by Eq. (5) because $v_{\mathrm{X}}=0$ at $P_{\text {add }} *$. Similarly, the second solid line in Fig. 7 was calculated from the second estimate of $K_{\mathrm{A}}\left(5.82 \times 10^{3} \mathrm{dm}^{3} \mathrm{~mol}^{-1}\right)$. Then, the second estimate of $P_{\text {add }} *$ was calculated as -0.065 psi from the second solid line in Fig. 7. The resulting values of $\mu_{1}$ and $K_{\mathrm{A}}$ was calculated as $1.13 \times 10^{3} \mathrm{~s}$ and $6.17 \times 10^{3} \mathrm{dm}^{3} \mathrm{~mol}^{-1}$, respectively.

Table 1 lists the results of the iterative calculations carried out in the same manner. The values of $K_{\mathrm{A}}, \mu_{1}$, and $P_{\text {add }} *$ were finally determined as $6.35 \times 10^{3} \mathrm{dm}^{3} \mathrm{~mol}^{-1}, 1.18 \times 10^{3} \mathrm{~s}$, and -0.082 psi, respectively. The third solid line in Fig. 7 corresponds to the final results, which is also plotted in Fig. 5. The two lines in Fig. 5 are approximately parallel, suggesting that $v_{\mathrm{S}}$ and $v_{\mathrm{X}}$ are affected by $P_{\text {add }}$ in the same manner. Figure 5 indicates that linear correlations are observed between $v_{\mathrm{S}}$ and $P_{\text {add }}$ and between $v_{\mathrm{X}}$ and $P_{\text {add }}$ and that they are almost parallel with each other. This means that the CE system was accurately pressurized with the same increment of $P_{\text {add }}, e . g .,-0.1,0$, and 0.1 psi. Additionally, in Fig. 6, the three solid triangles calculated from the final values of $K_{\mathrm{A}}, \mu_{1}$, and $P_{\text {add }} *$ are fairly overlaid on the open circles. The validity of their final values was demonstrated.

It should be noted that $P_{\text {add }} *(-0.082 \mathrm{psi})$ was larger than -0.1 psi. This means that $X$ migrates toward the inlet of the capillary at $P_{\text {add }}=-0.1$ psi. However, the elution peak of thymol was measured at $P_{\text {add }}=-0.1$ psi in Fig. 2 . Even at $P_{\text {add }}=-0.1$ psi, $S$ oppositely migrated to the outlet of the capillary and was detected. The CE system can be pressurized as long as $S$ can be detected even though $X$ migrates inversely to the inlet of the capillary. The value of $P_{\text {add }}$ is not a comprehensive physical parameter. It is necessary only for obtaining $K_{\mathrm{A}}$, then for determining $k_{\mathrm{a}}$ and $k_{\mathrm{d}}$. However, because $P_{\text {add }} *$ was located between -0.1 and $0 \mathrm{psi}, \mu_{1}$ and $\mu_{2}{ }^{\prime}$ could accurately be calculated by interpolating the correlations in Figs. 3 and 4.

\section{Determination of $k_{a}$ and $k_{d}$}

The value of $\mu_{2}{ }^{\prime}$ of thymol at $P_{\text {add }}{ }^{*}$ was estimated as $178 \mathrm{~s}^{2}$ from Fig. 3. The information about $D_{\mathrm{L}}$ is necessary for determining $k_{\mathrm{a}}$ and $k_{\mathrm{d}}$ because, as indicated in Eq. (3), $k_{\mathrm{a}} / k_{\mathrm{d}}{ }^{2}$ is obtained by subtracting the contribution of axial diffusion of thymol to $\mu_{2}^{\prime}$. As explained in the Supporting Information, it seems that the flow conditions in the CCE mode bring about an additional band broadening because $P_{\text {add }}$ is applied to the capillary. The value of $D_{\mathrm{L}}$ should be experimentally measured in the actual CE system containing SDS micelles. The value of $D_{\mathrm{L}}$ of DMSO was calculated as $2.7 \times 10^{-5} \mathrm{~cm}^{2} \mathrm{~s}^{-1}$ from $\mu_{2}^{\prime}$ at $P_{\text {add }} *$ in Fig. 4. According to the Wilke-Chang equation (refer to Supporting Information), ${ }^{28,29}$ this $D_{\mathrm{L}}$ value was corrected by considering the difference in the molar volume at a normal boiling point between thymol and DMSO. Consequently, $D_{\mathrm{L}}$ of thymol was estimated as $1.6 \times 10^{-5} \mathrm{~cm}^{2} \mathrm{~s}^{-1}$.

Then, the contribution of axial diffusion to $\mu_{2}{ }^{\prime}$ was calculated as $26 \mathrm{~s}^{2}$ from the estimated $D_{\mathrm{L}}$ value of thymol because $K_{\mathrm{A}}$ is already determined as $6.35 \times 10^{3} \mathrm{dm}^{3} \mathrm{~mol}^{-1}$. The value of $\mu_{2}{ }^{\prime}$ was 6.8 times larger than the contribution of axial diffusion. This means that the contribution of the reaction kinetics to $\mu_{2}{ }^{\prime}$ is 5.8 times larger than that of axial diffusion. An error in the estimation of the $D_{\mathrm{L}}$ value little influences the accuracy of the resulting values of $k_{\mathrm{a}}$ and $k_{\mathrm{d}}$. In conclusion, $k_{\mathrm{a}}$ and $k_{\mathrm{d}}$ for the intermolecular interaction between thymol and SDS micelle were appropriately determined as $5.6 \times 10^{4} \mathrm{dm}^{3} \mathrm{~mol}^{-1} \mathrm{~s}^{-1}$ and $8.7 \mathrm{~s}^{-1}$, respectively, by the MKCCE method.

\section{Chemical properties of SDS micelles}

It was reported for SDS micelle that the critical micelle concentration (CMC) was about $8 \mathrm{mmol} \mathrm{dm}^{-3}$ and the aggregation number was about 50 - 60 in water. ${ }^{1,30-35}$ However, the values of $\mathrm{CMC}$ and aggregation number of SDS micelle were experimentally measured because the run buffer used was a mixture of methanol and aqueous phosphate buffer. The CMC and aggregation number were measured as $2.1 \mathrm{mmol} \mathrm{dm}^{-3}$ and 49, respectively (refer to Supporting Information).

The initial concentration of SDS micelle, which was free in the run buffer, was calculated as $0.27(=(15.4-2.1) / 49) \mathrm{mmol}$ $\mathrm{dm}^{-3}$. In Fig. 2, the width of thymol peak at $P_{\text {add }}=+0.1$ and -0.1 psi was respectively about 30 and $75 \mathrm{~s}$ at about $1 \%$ peak height. The peak width was 6 and 15 times larger than that of solute injection pulse $(5.0 \mathrm{~s})$ under the conditions that thymol peak eluted fastest and slowest, respectively. An average concentration of thymol was calculated as 0.33 and $0.13 \mathrm{mmol}$ $\mathrm{dm}^{-3}$ because the concentration of thymol in the solute injection pulse was $2.0 \mathrm{mmol} \mathrm{dm}^{-3}$. It seems under equilibrium conditions that the amount of the thymol-SDS micelle complex was respectively calculated as 0.15 and $0.072 \mathrm{mmol} \mathrm{dm}^{-3}$ when $1: 1$ stoichiometry was assumed between them and that SDS micelles of 0.12 and $0.20 \mathrm{mmol} \mathrm{dm}^{-3}$ were free in the run buffer.

It is also probable that plural thymol molecules are solubilized by one SDS micelle. The number of solubilized azo dye molecules $\left(M_{\mathrm{w}}=232-247\right)$ per SDS micelle was reported as $1.7-2.1 .^{36}$ It was also reported that one SDS micelle solubilized $14-233$ molecules of $n$-fatty acids $\left(M_{\mathrm{w}}=144-228\right)$ and $8.9-$ 
209 molecules of $n$-higher alcohols $\left(M_{\mathrm{w}}=130-242\right)$, of which the carbon number in alkyl chains were $8-16 .{ }^{37}$ Although surfactants were not SDS, solubilization data of water insoluble dyes to micelles were also reported, ${ }^{38-40}$ i.e., 5 - 12 molecules of 1-o-tolyl-azo-2-naphthylamine were solubilized by one micelle of sodium dodecylpolyoxyethylene sulfates, ${ }^{38} 1.1-12$ molecules of $o$-tolyl-azo-2-naphthol were solubilized by one micelle of $\mathrm{N}$-alkyl betaines, ${ }^{39}$ and $1.6-4.0$ molecules of $\mathrm{N}$-dimethylaminoazobenene were solubilized by one micelle of polyoxyethylene nonylphenols. ${ }^{40}$ It is probable that the CE data in this study can be analyzed by assuming that $C_{\mathrm{L}}$ is substantially equal to $0.27 \mathrm{mmol} \mathrm{dm}^{-3}$ because of the solubilizing capacity of SDS micelle. In addition, Figs. 5 - 7 indicate that the experimental data of $\mu_{1}$ measured under the different conditions of $P_{\text {add }}$ are consistently explained by Eqs. (2) and (5). This means that $C_{\mathrm{L}}$ can be regarded substantially constant at $0.27 \mathrm{mmol} \mathrm{dm}^{-3}$.

\section{Advantageous characteristics of the MKCCE method}

The MKCCE method has some advantageous features as follows (more detailed explanations are provided in Supporting Information).

It is not required to immobilize ligand molecules on a support. The MKCCE method is a label-free method. No chemical modification, e.g., fluorescence labeling, of solute and/or ligand molecules is required. The MKCCE method is suitable for the analysis of weak intermolecular interactions. As indicated in Eq. (2), the value of $K_{\mathrm{A}}$ depends on that of $C_{\mathrm{L}}$. When the minimum value of the product, i.e., $K_{\mathrm{A}} C_{\mathrm{L}}$, is assumed to be 0.01 in comparison with $1, K_{\mathrm{A}}$ is calculated as 0.1 and $0.01 \mathrm{dm}^{3} \mathrm{~mol}^{-1}$ at $C_{\mathrm{L}}=0.1$ and $1 \mathrm{~mol} \mathrm{dm}^{-3}$. Under such conditions, a sufficient amount of ligand must be presented in the capillary. The basic principle for analyzing intermolecular interactions is different between the MKCCE method and the other methods. All compounds can be regarded as solute or ligand irrespective of their chemical properties as long as they are treated in CE experiments. The data analysis procedure is quite easy because moment equations are mathematically simple. The values of $k_{\mathrm{a}}$ and $k_{\mathrm{d}}$ can be analytically determined by the moment theory from experimental CE peaks. The MKCCE method requires no special experimental skill. CE instruments commercially available can be used with no special modification. The wasteful use of reagents can be suppressed.

As described above, the MKCCE method has some advantages over other methods developed for the kinetic study of intermolecular interactions, e.g., surface plasmon resonance and fluorescence cross-correlation spectroscopy. On the other hand, the KCE method similarly has most of the advantages because it is also based on CE measurements. However, in the case of the MKCCE method, it is unnecessary to simulate elution curves by numerical calculations and to fit them to experimental data in order to determine the values of $k_{\mathrm{a}}$ and $k_{\mathrm{d}}$. They can be analytically determined from experimental CE peaks by the MKCCE method. It is expected that the values of $k_{\mathrm{a}}$ and $k_{\mathrm{d}}$ are more accurately determined by the MKCCE method than the KCE method.

\section{Conclusions}

The CCE concept with the moment analysis theory was introduced to improve the performance of $\mathrm{CE}$ for the kinetic study of intermolecular interactions. However, because it was difficult to really perform CE experiments under the CCE conditions, we also developed the practical experimental and data analysis procedures to determine $k_{\mathrm{a}}$ and $k_{\mathrm{d}}$. The rate constants $\left(k_{\mathrm{a}}\right.$ and $\left.k_{\mathrm{d}}\right)$ of the interaction in the MEKC system between thymol and SDS micelle were analytically determined from the $\mu_{1}$ and $\mu_{2}^{\prime}$ values of CE peaks measured. The introduction of the CCE concept made it possible to analytically determine $k_{\mathrm{a}}$ and $k_{\mathrm{d}}$ from CE peaks by the moment theory. It is unnecessary to fit elution curves numerically calculated to experimental data for determining $k_{\mathrm{a}}$ and $k_{\mathrm{d}}$. It is concluded that the MKCCE method is effective for the kinetic study of intermolecular interactions. The MKCCE method has some advantageous characteristics for the study of affinity kinetics. It is required to acquire more analytical results for the kinetic study of chemical and biological affinity interactions to demonstrate the effectiveness of the MKCCE method. This is the subject under investigated.

\section{Acknowledgements}

This study was, in part, supported by the MEXT-Supported Program for the Strategic Research Foundation at Private Universities and by the Rikkyo University Special Fund for Research 2015.

\section{Supporting Information}

Some items of additional information are provided for the derivation of the moment equations for the analysis of electropherograms measured in the CCE mode, the experimental conditions and data analysis procedures, the correction of the contribution of axial diffusion to $\mu_{2}{ }^{\prime}$, the chemical properties of SDS micelles, and some advantageous characteristics of the MKCCE method. These materials are available free of charge on the Web at http://www.jsac.or.jp/analsci/.

\section{References}

1. L. J. Cline Love, J. G. Habarta, and J. G. Dorsey, Anal. Chem., 1984, 56, 1132A.

2. S. A. Shamsi, C. P. Palmer, and I. M. Warener, Anal. Chem., 2001, 73, 140A.

3. I. J. Colton, J. D. Carbeck, J. Rao, and G. M. Whitesides, Electrophoresis, 1998, 19, 367.

4. Z. Chen and S. G. Weber, TrAC, Trends Anal. Chem., 2008, 27, 738

5. C. Jiang and D. W. Armstrong, Electrophoresis, 2010, 31, 17.

6. S. Kiessig, A. Stettler, S. Fuhrimann, and M. A. Schwarz, "Supramolecular Chemistry: From Molecules to Nanomaterials", 2012, John Wiley and Sons, New York.

7. C. D. Newman and G. E. Collins, Electrophoresis, 2008, $29,44$.

8. L. Z. Avila, Y.-H. Chu, E. C. Blossey, and G. M. Whitesides, J. Med. Chem., 1993, 36, 126.

9. A. Bromberg and R. A. Mathies, Anal. Chem., 2003, 75, 1188.

10. S. N. Krylov, Electrophoresis, 2007, 28, 69.

11. V. A. Galievski, A. S. Stasheuski, and S. N. Krylov, Anal. Chem., 2015, 87, 157.

12. V. Okhonin, S. M. Krylova, and S. N. Krylov, Anal. Chem., 2004, 76, 1507.

13. A. Petrov, V. Okhonin, M. Berezovski, and S. N. Krylov, J. Am. Chem. Soc., 2005, 127, 17104.

14. G. G. Mironov, V. Okhonin, S. I. Gorelski, and M. V. 
Berezovski, Anal. Chem., 2011, 83, 2364.

15. V. Okhonin, M. V. Berezovski, and S. N. Krylov, J. Am. Chem. Soc., 2010, 132, 7062.

16. L. T. Cherney and S. N. Krylov, Anal. Chem., 2011, 83, 1381.

17. K. Miyabe and G. Guiochon, J. Phys. Chem. B, 2002, 106, 8898.

18. K. Miyabe, Anal. Chem., 2007, 79, 7457.

19. K. Miyabe, J. Chromatogr. A, 2008, 1183, 49.

20. K. Miyabe, Anal. Sci., 2011, 27, 1007.

21. K. Miyabe, J. Chromatogr. A, 2014, 1356, 171.

22. Y. Daneshbod, J. D. Sterling, and A. Nadim, Phys. Rev. E, 2007, 76, 051922.

23. Z. Deyl and F. Svec, "Capillary Electrochromatography", 2001, Elsevier Science, Amsterdam.

24. R. H. H. Neubert and H.-H. Ruttinger, "Affinity Capillary Electrophoresis in Pharmaceutics and Biopharmaceutics", 2003, Marcel Dekker, New York.

25. J. P. Landers, "Handbook of Capillary and Microchip Electrophoresis and Associated Microtechniques", 2008, CRC Press, Taylor \& Francis Group, Boca Raton.

26. L. Müllerová, P. Dubský, J. Svobodová, and B. Gaš, Electrophoresis, 2013, 34, 768.

27. A. Hellqvist, Y. Hedeland, and C. Pettersson, Electrophoresis,
2013, 34, 3252.

28. B. E. Poling, J. M. Prausnitz, and J. P. O'Connel, "The Properties of Gases and Liquids", 2001, McGraw-Hill, New York.

29. R. E. Treybal, "Mass-Transfer Operations", 1980, McGrawHill, New York.

30. D. A. Doughty, J. Phys. Chem., 1979, 83, 2621.

31. P. Lianos and R. Zana, J. Phys. Chem., 1980, 84, 3339.

32. S. S. Berr and R. M. Jones, Langmuir, 1988, 4, 1247.

33. M. H. Gehlen and F. C. De Schryver, J. Phys. Chem., 1993, 97, 11242 .

34. F. H. Quina, P. M. Nassar, J. B. S. Bonilha, and B. L. Bales, J. Phys. Chem., 1995, 99, 17028.

35. B. L. Bales, L. Messina, A. Vidal, M. Peric, and O. R. Nascimento, J. Phys. Chem. B, 1998, 102, 10347.

36. M. Abe, N. Suzuki, and K. Ogino, J. Colloid Interface Sci., 1984, 99, 226.

37. K. Ogino, M. Abe, and N. Takesita, Bull. Chem. Soc. Jpn., 1976, 49, 3679.

38. F. Tokiwa, J. Phys. Chem., 1968, 72, 1214.

39. J. Swarbrick, J. Galownia, and T. R. Bates, J. Colloid Interface Sci., 1972, 41, 609.

40. K. S. Birdi, Colloid Polym. Sci., 1974, 252, 551. 